\title{
Occupational exposure to sharps injuries among doctors working in two Teaching Hospitals in the Sothern Province
}

\author{
Wimalasena $\mathrm{TB}^{1}$, Liyanarachchi $\mathrm{ND}^{2}$, Wijesinghe $\mathrm{CJ}^{2}$, De Silva $\mathrm{PV}^{2}$
}

\begin{abstract}
Introduction: Sharps injuries with contaminated instruments are a common occupational hazard among health care workers, often exposing them to life threatening blood borne infections. Management of sharps injuries poses a considerable burden on health care system, due to expensive post exposure investigations and treatment. This study was conducted to assess the incidence and post-exposure management of sharps injuries among doctors working in two teaching hospitals in Southern Province.
\end{abstract}

Methods: A hospital based, cross-sectional study was conducted among a random sample of 248 doctors working in Teaching Hospitals Karapitiya and Mahamodara. Data on exposure to sharp injuries and post-exposure management were collected using a selfadministered questionnaire and analyzed using SPSS software package. Ethical approval for the study was obtained from the Ethical Review Committee, Faculty of Medicine, University of Ruhuna.

Results: The sample consisted of 42 (16.9\%) consultants, 33 (13.3\%) postgraduate trainees, 148 (59.7\%) grade medical officers and $25(10.1 \%)$ interns. More than one fifth $(n=53,21.5 \%)$ of the sample have sustained sharp injuries during patient care encounters during previous 6 months. Out of them, $94.3 \%(n=50)$ were needle prick injuries. Almost two thirds $(n=34,64.2 \%)$ of these sharp injuries were not notified to the infection control unit of the respective hospital. Risk assessment was done in only $68.4 \%$ of notified cases. A statistically significant association was found between exposure status and the professional category of doctors. Intern medical officers reported the highest percentage of sharps injuries, whereas the consultants were the least exposed $(68 \%$ Vs. $2.4 \%, p<0.01)$.

Conclusions and recommendations: Exposure to sharp injuries is a common occurrence among doctors. Professional category is associated with risk of exposure and the current post-exposure management is highly unsatisfactory. Training on standard precautions, particularly on prevention and management of sharps injuries should be emphasized more in medical curricula.

1. Ministry of Health

2. Faculty of Medicine, University of Ruhuna
Periodic ongoing training programmes should be conducted on above practices.

\section{Introduction}

Hospital Associated Infections (HAI) is recognized as a serious adverse event of health care delivery around the world and is one of the major occupational hazards encountered by health care workers (1). HAl is defined as 'an infection occurring in a patient during the process of care in a hospital or other health care facility which was not present or incubating at the time of admission' (1).

According WHO statistics, occupational exposure to $\mathrm{HAl}$ among health care workers $(\mathrm{HCW})$ is a common occurrence (2). Out of 35 million HCW worldwide, around three million gets percutaneous exposure to blood born infectious agents every year, exposing two million to HBV, 0.9 million to HCV and 750000 to HIV infections. This may result in 15,000 HCV, 70,000 HBV and 1000 HIV infections, posing a serious burden on health care systems. More than $90 \%$ of these affect HCWs in developing countries. All doctors have high risk of getting exposed to blood and other body fluids while attending to patients as they have immediate contact with patients. Percutaneous injuries like needle stick or other sharps injuries and splash of blood or other body fluids into mucus membranes are the possible modes of acquisition of HAl among doctors. In most of these situations, the exposure occurs through a needle prick injury or cut with a sharp instrument contaminated with blood (3) A study conducted in Colombo, Sri Lanka revealed that over $1 / 5^{\text {th }}$ of the doctors had some form of exposure to sharp injuries, making them vulnerable for HAI (4). Similar findings are observed in many other settings $(5,6,7)$.

Sharps injuries with objects or needles contaminated with blood could not only expose doctors to pathogens causing life-threatening infections, but also lead to expensive post exposure investigations and treatment. Health care systems of developing countries like Sri Lanka cannot afford to allocate limited resources available on management of the consequences. On the other hand, occurrence of these events is largely preventable. Hence, hospitals should have policies and protocols to minimize occupational exposure among HCW. 
Prevention of exposure to blood-borne pathogens can be achieved by correct handling of sharps by HCW. According to the guidelines given in Hospital Infection Control Manual of the Sri Lanka College of Microbiologists, avoiding use of sharps whenever possible and using scalpels, needles and syringes as single use items would reduce the risk of sharp injuries (8). In addition, care should be taken in handling, cleaning and disposing sharps. Used needles should not be recapped with two hands and bending or breaking of used needles by hand should be avoided. Sharps should be discarded immediately after use, directly into a sharps bin which is placed close to point of use. Passing sharps from hand to hand should not be done.

Despite all the precautions taken, occupational exposure to sharps injuries by contaminated instruments does occur sometimes. Therefore, there should be appropriate post exposure management guidelines and protocols for prompt reporting, evaluation, counseling and treatment. In managing a health care worker potentially exposed to HBV or HIV, care of the exposure site, reporting to infection control team and assessment of the risk associated with exposure should be done. Expose source should be tested for HBV, HCV and HIV antibody. Exposed person should be evaluated and Hepatitis B immunoglobulin and antiretroviral drugs should be given if indicated (8). The occupational exposures should be followed up after initial treatment as the exposed HCWs have a risk of acquiring infection (9). A periodic assessment of exposure to sharp injuries among doctors, as well as identifying the factors that pre-dispose to such injuries are two important aspects that will be useful in monitoring this process. Therefore, this study was carried out with the aim of assessing the incidence and post-exposure management of sharp injuries among doctors working in the two teaching hospitals in Southern Province.

\section{Methods}

This study was a hospital based, cross sectional survey, carried out as a component of a larger study assessing knowledge and adherence to Standard Precautions among doctors. The study was conducted in the Teaching Hospital, Karapititya (THK) and Teaching Hospital Mahamodara (THM). THK is the largest tertiary care hospital in the Southern Province, with bed strength of 1711. It has OPD, ETU, ICU operating theatres, wards, with all major and minor specialities except obstetrics and gynaecology. During the study period 593 doctors were working in THK. THM is the only tertiary care maternity hospital of Southern Sri Lanka. This hospital provides gynaecology, obstetrics and neonatology services. Bed strength is 375 and during the study period 90 doctors worked in this hospital. The study included a sample of 248 doctors working in THK and THM, including consultants (specialist grade medical officers), senior registrars, registrars, grade medical officers and intern medical officers. Doctors who have worked less than six months in these hospitals and those who were on leave during the study period were excluded from the sample. A stratified random sampling method was followed to include doctors representing each professional category. The number of doctors from each category was proportionate to its distribution among the total study population.

A pre-tested, self-administered questionnaire was used to assess the incidence of sharp injuries among doctors during the last 6 months and other relevant variables. The collection of data and all observations were carried out by the principal investigator himself to prevent interobserver variation. Informed consent was obtained from the Ethical approval for the study was obtained from the Ethical Review Committee of the Faculty of Medicine, University of Ruhuna and permission was obtained from the two directors of THK and THM before the data collection. Data collection was done for one month period from 01.09.2013 to 30.09.2013 and collected data were analysed using statistical Package for Social sciences (SSPS) version 17.0.

\section{Results}

The overall response rate in the sample was very good (91.9\%). Out of the participants, 211 (85.1\%) were from THK and 37 (14.9\%) were from THM. Majority were grade medical officers ( $n=148,59.7 \%)$. The highest number of respondents were from medical wards $(n=68$, $27.4 \%$ ), the least number representing the obstetrics and gynaecology wards ( $n=15,6.0 \%)$. The characteristics of the study sample are described in Table 1.

More than one fifth out of 246 doctors ( $n=53,21.5 \%$ ) have sustained sharp injuries during patient care encounters in the period of last 6 months (Table 2). Out of those who had sharp injuries, $94.3 \%$ were needle prick injuries. Almost two thirds of these sharps injuries $(n=34,64.2 \%)$ were not notified to the infection control unit of the respective hospital. Risk assessment was done in only $13(68.43 \%)$ of the notified cases. Bivariate analysis was performed to assess whether there is a difference between proportions of sharps injuries according to professional categories of doctors, type of unit they are working, the duration of service and the training received on Standard Precautions (Table 3). Intern medical officers had the highest percentage of sharps injuries (68\%), followed by registrars and senior registrars (24.2\%). Consultants had the least amount of sharps injuries (2.4\%). The difference in the proportion of sharp injuries among categories of doctors was highly significant $(p<0.01)$. Similarly, the doctors working in 
surgical wards were more likely to sustain sharp injuries compared to those working in other units $(p<0.05)$. Service duration of less than 5 years also demonstrated a significant association with sharp injuries $(p<0.001)$. Although training on Standard Precautions received during undergraduate period was not associated with history of sharp injuries, those who had training after graduation reported significantly reduced exposure to these events $(<0.001)$.

\section{Discussion}

Management of sharps is an important aspect of standard precautions, designed to prevent hospital associated infections. In this study, we were able to identify several aspects of sharps management that expose the doctors to an increased risk of infections. The fact that more than one fifth $(n=53,21.5 \%)$ of doctors in THK and THM have had sharps injuries during a period of six months is a very important finding of this study. Out of those $94.3 \%$ were needle prick injuries. In comparison, a study done among HCWs in four Teaching Hospitals of Colombo district revealed that $23.1 \%$ doctors have had prick with a needle contaminated with blood and $8.5 \%$ of doctors have had a cut with sharp instrument contaminated with blood (4). Victims of sharp injuries seem to be greater among individuals under training, as revealed in a survey done among 4th year medical students of university of Colombo. This study reported that $95 \%$ of the students had sustained one or more sharp injury. Out of these, $89 \%$ has occurred during suturing, $23 \%$ during venepuncture and $14 \%$ while assisting in deliveries (10). These findings suggest that sharp injuries continue to remain a considerable hazard among hospital settings in Sri Lanka. Evidence from other settings reports higher incidence of sharps injuries among doctors, especially in the regional countries. In an observational study conducted in Pakistan to assess the impact of a surveillance and educational program on the rate of needle stick injuries among HCWs, junior doctors were reported to have sustained the highest number of injuries (28.5\%). Nearly $1 / 5^{\text {th }}$ of sharp injuries were reported during blood collection (5). A research done in India has shown that needle stick injuries were reported by $50 \%$ of nurses, $31 \%$ of doctors and $25 \%$ of interns. Recapping was found to be the commonest cause reported by $68 \%$ of nurses $40 \%$ of doctors and $52 \%$ of interns (6). A Nigerian research revealed that $16.87 \%$ of doctors have experienced needle pricks during a period of 3 months, which indicates a higher incidence than our study (11). In a study done in Germany, $47 \%$ of physicians were found to have at least one needle prick injury per year (12). A similar study done in UK reported that $37 \%$ of participants had needle pricks during a period of one year (7). These finding are closely comparable to the results of our study. However, a lower incidence of needle stick injuries had been reported among health care workers in other settings such as Australia and Italy (3). In keeping with our findings, needle prick injuries were the commonest among sharp injuries in several other studies $(5,3)$. The other component of this study assessing the knowledge and practices related to Standard Precautions revealed interesting findings that can explain these risk exposures (13). The practices of handling sharps among the doctors in the study sample revealed that a considerable proportion of doctors had done 'bending of needle' during episiotomy suturing (75\%) and 'recapping' during venepuncture (30-33\%), which could lead to needle stick injuries.

In this study we observed that there is an association between the exposure to sharp injuries and the professional category, reporting the highest percentage of sharps injuries among intern medical officers. However, studies from other settings show contrasting evidence. A Pakistan study concluded that the junior doctors have sustained the highest number of sharps injuries (5), whereas, an Indian study revealed a higher rates of needle stick injuries among residents compared with interns (6). The knowledge, attitudes and practice of doctors in different settings could be resulting in different outcomes so that direct comparison of these rates may not be appropriate. Although one could argue that the junior doctors are more likely to be exposed to these injuries than the consultants because they are more involved in day to day patient care activities, we cannot exclude the possibility of having more incorrect practices probably as a result of poor awareness and less practical experience among this group. If awareness and adherence to standard precautions is optimal, this type of injuries could not have occurred, despite being exposed to more opportunities. The association between sharp injuries and training on Standard Precautions after graduation further supports this assumption. Almost two thirds (64.2\%) of sharps injuries sustained by doctors were not notified to infection control unit. This very clearly indicates the lack of awareness on post-exposure management of sharp injuries among doctors. Galle is an area where tourism is blooming up and this may increase the risk of health care workers' contact with HIV patients. Therefore, poor awareness on prevention of sharps injuries and post exposure management could be a problem in the future.

Although the results of this study could be affected by recall bias in reporting sharps injuries and the postexposure management, it would have resulted in underreporting of the incidence. The relatively high incidence of sharps injuries observed could not have been explained by this limitation. 


\section{Conclusions and recommendations}

In conclusion, this study revealed that more than one fifth $(21.5 \%)$ of doctors have sustained sharps injuries during a period of six months and the reporting of sharps injuries is poor.

In order to prevent these hazardous exposures, an organizational culture should be created where adherence to standard precautionary guidelines is an integral part of giving high quality patient care. Doctors should be educated and persuaded to notify sharps injuries without delay. Medical under graduate curricula could place more emphasis on teaching standard precautions and infection control practices. Mandatory training on standard precautions needs to be included in the orientation program of intern medical officers. Periodic ongoing reorientation training programs should be arranged for all categories of doctors. Infection control team should play an active role in training and interacting with doctors, motivating and exerting positive influences on their practices. The consultants and senior registrars can act as role models to influence the practices of peers and junior doctors, especially in areas like proper sharps handling.

\section{Acknowledgements}

The authors wish to thank the Directors of Teaching Hospital Karapitiya and Teaching Hospital Mahamodara, DrAjith Nagahawatte, Senior Lecturer in Microbiology

\begin{tabular}{|c|c|c|c|}
\hline & Characteristic & No & $\%$ \\
\hline \multirow[t]{2}{*}{ Institution } & TH, Karapitiya & 211 & 85.1 \\
\hline & TH, Mahamodara & 37 & 14.9 \\
\hline \multirow[t]{2}{*}{ Gender } & Male & 115 & 46.4 \\
\hline & Female & 133 & 53.6 \\
\hline \multirow[t]{4}{*}{ Age $(n=245)$} & 30 yrs or less & 41 & 16.7 \\
\hline & $31-40$ yrs & 104 & 42.4 \\
\hline & $41-50$ yrs & 84 & 34.3 \\
\hline & above 50 yrs & 16 & 6.5 \\
\hline \multirow[t]{4}{*}{ Professional category } & Consultants & 42 & 16.9 \\
\hline & Registrars / Senior Registrars & 33 & 13.3 \\
\hline & Grade medical Officers & 148 & 59.7 \\
\hline & Inter Medical Officers & 25 & 10.1 \\
\hline \multirow[t]{7}{*}{ Unit of work } & Intensive Care Unit & 37 & 14.9 \\
\hline & Medical wards & 68 & 27.4 \\
\hline & Surgical wards & 50 & 20.2 \\
\hline & Paediatric wards & 27 & 10.9 \\
\hline & $\begin{array}{l}\text { Obstetrics \& Gynaecology } \\
\text { wards }\end{array}$ & 15 & 6.0 \\
\hline & Out Patient Department & 17 & 6.9 \\
\hline & Other & 34 & 13.7 \\
\hline \multirow[t]{4}{*}{ Duration of service $(n=245)$} & 5 yrs or less & 68 & 27.8 \\
\hline & $6-10$ yrs & 66 & 26.9 \\
\hline & $11-15$ yrs & 45 & 18.4 \\
\hline & $16-20 \mathrm{yrs}$ & 44 & 18.0 \\
\hline
\end{tabular}


Table 2: Details about sharp injuries sustained by doctors in the sample during the last 6 months and post-exposure management

Details about sharp injuries

Sustained sharp injury/injuries during last 6 months

( $n=246)$

Yes

No

Type of injury $(n=53)$

Cut injury

Needle prick injury

Notified the injury to infection control unit $(n=53)$

Yes

No

Type of post-exposure management $(n=19)$

Counseling

Risk Assessment

$\%$

1.5

5

7 


\section{References}

1. World Health Organization. Prevention of hospital acquired infections A PRACTICAL GUIDE : WHO 2002 (online). retrieved on 03.10.2015

2. World Health Organization. Healthcare worker safety AIDE_MEMORIE for a strategy to protect health workers from infections with blood borne viruses.

3. Mallon DF, Shearwood W, Mallal SA, French MA, Dawkins RL. Exposure to blood borne infections in healthcare workers of a major teaching hospital in Australia. Medical Journal of Australia, 1992;159 (9): 92-95.

4. Karavita DA, Gunathilaka MP, Hettiarachchi I, Ariyaratne KAM, Ariyaratne L, Abeywardena MLDS. The knowledge practices and the extent of occupational exposure to blood among health care workers in Colombo. A cross sectional study. Sri Lanka Journal of Veneriology, 2012;3 (1):23-28.

5. Zafa A, Habib F, Hadwani R, Ejaz M, Khowja K, Khowja $R$, Infan S. Impact of infection control activities on the rate of needle stick injuries at a tertiary care hospital of Pakistan over a period of six years:an observational study. BMC Infectious Diseases 2009:9 (78).

6. Payghan BS, Kadam SS, Kumar P, Sridevi BK. Knowledge and perceptions of health care workers towards clean care practices in a tertiary care hospital. Journal of Evolution of Medical and Dental Sciences, 2013; 2 (10) $1459-60$.

7. Stein AD, Marakawo TP. A survey of doctors' and nurses' knowledge, attitudes and compliance with infection control guidelines in Birmingham Teaching Hospitals. Journal of Hospital Infection, 2003; 54 (1).
8. Hospital Infection Control Manual. Sri Lanka College of Microbiologists. 2005

9. Centre for Disease Control and Prevention Healthcare associated infections :CDC 2011 . http:// www.cdc.gov/HAl/organisms/hiv/SurveillanceOccupationally-Acquired-HIV-AIDS.html [accessed on 03.10.2015]

10. Liyanage IK, Caldera TSKRD, Rajapaksha RWMA, Liyanage CK, De Silva P, Karunathilaka IM. Sharp injuries among medical students in the Faculty of Medicine Colombo, Sri Lanka. International Journal of Occupational Medicine and Environmental Health, 2012; 25 (3):275 - 80.

11. Okechukwu EF, Modetshi C. Knoeledge and practice of standard precautions in public health facilities in Abuja, Nigeria. International Journal of Infection Control, 2012; 8 (13).

12. Wirker S, Jang J. Alwinn R, GoHschalk R, Rabenan HF. Prevalence and prevention of needle stick injuries among health care workers in a German University Hospital. Internal Archives of Occupational and Environmental Health, 2008; 81.347-54.

13. Wimalasena TB, Wanninayake WMIK, Fernando GHS, Somaratne CJK, Chandrasena APRS, De Silva PV. Standard Precautions to reduce hospital associated infections: Knowledge and practices of doctors working in two Teaching Hospitals of Southern Sri Lanka. Journal on Health Policy and Management; 2 (In press) 